\title{
Tool for Power Factor Analysis and Passive Solution Design for Industrial Plants using Fuzzy Inference
}

\author{
Fredy H. Martínez S. ${ }^{\# 1}$, Fernando Martínez S. ${ }^{\# 2}$ and Edwar Jacinto G. ${ }^{\# 3}$

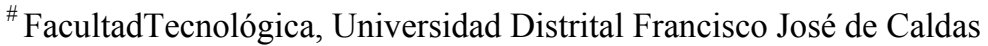 \\ Carrera 13 \#40-53, Bogotá D.C., Colombia \\ ${ }^{1}$ fhmartinezs@udistrital.edu.co \\ ${ }^{2}$ fmartinezs@udistrital.edu.co \\ 3 ejacintog@udistrital.edu.co
}

\begin{abstract}
This paper presents a tool developed to support the design of solutions to the problem of reactive power in industrial installations. From power data taken from the electrical installation (Watts [W], Volt-Ampere [VA], Volt-Ampere reactive [VAr] and Power Factor [PF]), the tool draws and analyses the historical behaviour, and proposes a power factor correction solution. The tool also calculates the minimum, average and maximum reactive power required by the system. With this limit data the software analyses the power factor correction behaviour and specifies the reactive power for a 6step mixed compensation bank (power per step, including the fixed step). The performance of the tool is evaluated on a data set recorded with a power analyser in a real industrial plant.
\end{abstract}

Keyword - AC power, Active power, Apparent power, Compensation bank, Fuzzy inference, Industrial plant, Power Factor, Volt-Ampere

\section{INTRODUCTION}

Any industrial plant with an intense use of electric motors in its processes has problems of poor PF. A low PF in an industrial plant is synonymous of voltage regulation problems, oversized electrical equipment, underutilization of installed electrical capacity, and even economic penalties from power supply utilities [1,2]. Although the solution to the problem requires a significant investment, it is well known that the solution of poor $\mathrm{PF}$ is not only economic but also profitable in comparison to costs [3].

In short, $\mathrm{PF}$ in the presence of linear loads corresponds to the ratio of active power $(P)$ to apparent power $(S)$. This phase shift can be measured as the phase shift between voltage and current signals $(\cos \phi)$, and produces additional reactive power $(Q)$ that requires higher generation, transmission and utilization costs of the useful electrical power. This problem can be theoretically solved by adjusting the phase difference between voltage and current $[4,5]$, however, it is normal to use a capacitor bank that compensates for the connected reactive power, normally generated by electrical machines [6].

During the last 30 years, the percentage of non-linear loads in the electrical power grid has been progressively increasing. This trend is due to the increase in grid-connected electronic equipment, particularly at the residential and commercial levels. Although these loads in the early years were small compared to industrial loads, the current massive increase in non-linear loads makes them a real problem of energy quality. In addition, at the industrial level, electronic drives for engines make the situation more complex [7]. In industrial plants this problem is mixed with the traditional inductive excess load, generating additional problems during the switching of the reactive banks [8]. These cases can be treated by the installation of passive harmonics in derivation or passive harmonic filters if the cost/benefit so justifies, or when there is a possibility of resonance with the capacitor bank. On this design, some strategies of design of the filters have been postulated in order to reduce to the maximum their cost [9].

In cases of high levels of harmonic distortion, the first step is always to try to relocate the loads to minimize both the PF and the harmonic level $[10,11]$. Then a network model is made that allows its analysis and simulation for different scenarios, in which both the use of filters and capacitor banks are considered [12].

Once the problem of harmonic distortion is eliminated, the PF must be corrected by proper installation of reactive power. This power, usually of the capacitive type, is installed with banks of certain sizes and quantities. The selection of the steps to be installed depends on the analysis of the installed load [13]. In any case, a control strategy capable of making switching decisions based on real-time readings of power on the network is required $[14,15,16]$. 
It is normal that the selection of the quantity and capacity of the capacitor banks is made to trial and error from the experience of the designer, however, there are proposals to optimize this design [17]. This paper proposes a software tool to support the design of passive PF correctors. The tool, based on a dataset registered with a power analyzer in an industrial plant, will be able to graph and support the power and PF analysis.

The following part of the paper is arranged in this way. Section 2 presents preliminary concepts and problem formulation. Section 3 illustrates the design profile and development methodology. Section 4 we present the preliminary results. And finally, in Section 5, we present our conclusions.

\section{Problem Formulation}

The problem of poor PF requires the study of two problems: Displacement PF and Total PF. Displacement PF corresponds to the case where linear loads prevail in the electrical installation, and the problem is mainly due to the phase shift between the voltage and current signals (Equ. 1). In the second case, the installation has a significant number of non-linear loads that produce currents and distortion powers, which implies solving the problem by first reducing the harmonic content.

$$
P F=\frac{P}{S}=\cos \phi
$$

Before selecting a solution to the problems of low PF, an evaluation of the harmonic content of the network is necessary. If it is too high, it is necessary to minimize it before installing a capacitor bank. According to EN 61800-3 the most important parameters for harmonic content analysis in an industrial plant with a large number of engine drives are $T H D_{i}, T D R_{i}, I D R_{i}$, and $S_{S T}$. These parameters are defined below assuming that $I$ is the total current, $I_{1}$ is the fundamental harmonic current, $I_{\mathrm{h}}$ is the $h$ harmonic current, $U_{N}$ is the nominal voltage, and $I_{T N}$ is the equivalent reference current.

The Total Harmonic Distortion $\left(T H D_{i}\right)$ is defined as (Equ. 2):

$$
\text { THD }_{i}=\frac{\sqrt{\sum_{h=2}^{H}\left(I_{h}\right)^{2}}}{I_{1}}(2)
$$

The Total Distortion Ratio $\left(T D R_{i}\right)$ is defined as (Equ. 3):

$$
T D R_{i}=\frac{\sqrt{I^{2}-I_{1}^{2}}}{I_{1}}(3)
$$

The Individual Distortion Ratio $\left(I D R_{i}\right)$ is defined as (Equ. 4):

$$
I D R_{i}=\frac{I_{h}}{I_{1}}(4)
$$

And the Subscribed Apparent Power $\left(S_{S T}\right)$ is defined as (Equ. 5):

$$
S_{S T}=\sqrt{3} \cdot U_{N} \cdot I_{T N}(5)
$$

This analysis is performed by measuring the variables in a point of common coupling, or in an internal point of coupling. Once the problem of harmonic content has been eliminated, an analysis of the behaviour of the network must be made for the design of a passive solution (selection of the reactive power compensation bank).

The goal is to develop a software tool to support the design of passive PF correctors. The tool, based on a dataset registered with a power analyzer in an industrial plant, will be able to graph and support the power and $\mathrm{PF}$ analysis, as well as set values for the minimum, average and maximum reactive power required by the system. With this data it should facilitate the analysis of PF correction behaviour and specify the reactive power for a 6-step mixed compensation bank (power per step including the fixed step).

\section{Methodology}

The aim of the tool is to support the design of passive solutions for poor PF in industrial plants. To do this, the tool must work with plant operation data corresponding to P, S, VAr and PF. The dataset must correspond to representative work cycles of the plant, and at the moment of its use the possible problem of harmonics must have been eliminated, either by verifying that its incidence is minimal or by using some type of filter to attenuate its effect.

This information (P, S, VAr and PF) must be used to define minimum, average and maximum values of reactive power in the installation. This can be done in many ways, the software allows the designer to play with these values, but also proposes some from the segmentation of the reactive power. This data will be used to analyze the PF behavior and specify the reactive power for a six-step mixed compensation bank (power per step, including the fixed step). 
The first window of the software facilitates the capture of the dataset and its visualization (Fig. 1). The file is loaded with the upper left button. The dataset must be in Microsoft Excel format, and organized by columns (Fig. 2 ). With the buttons at the bottom right it is possible to graph the information. On the left side are fields that allow the designer to evaluate different values (low, medium and high) for reactive power and PF.

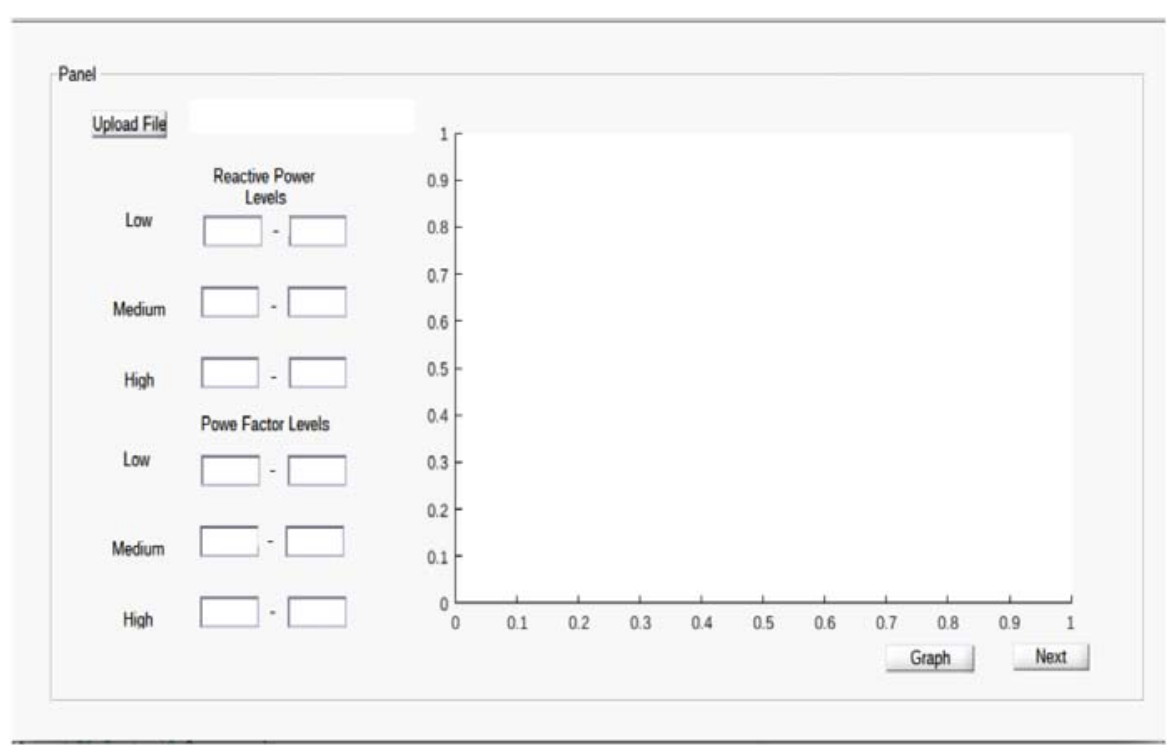

Fig. 1.Data capture and display window

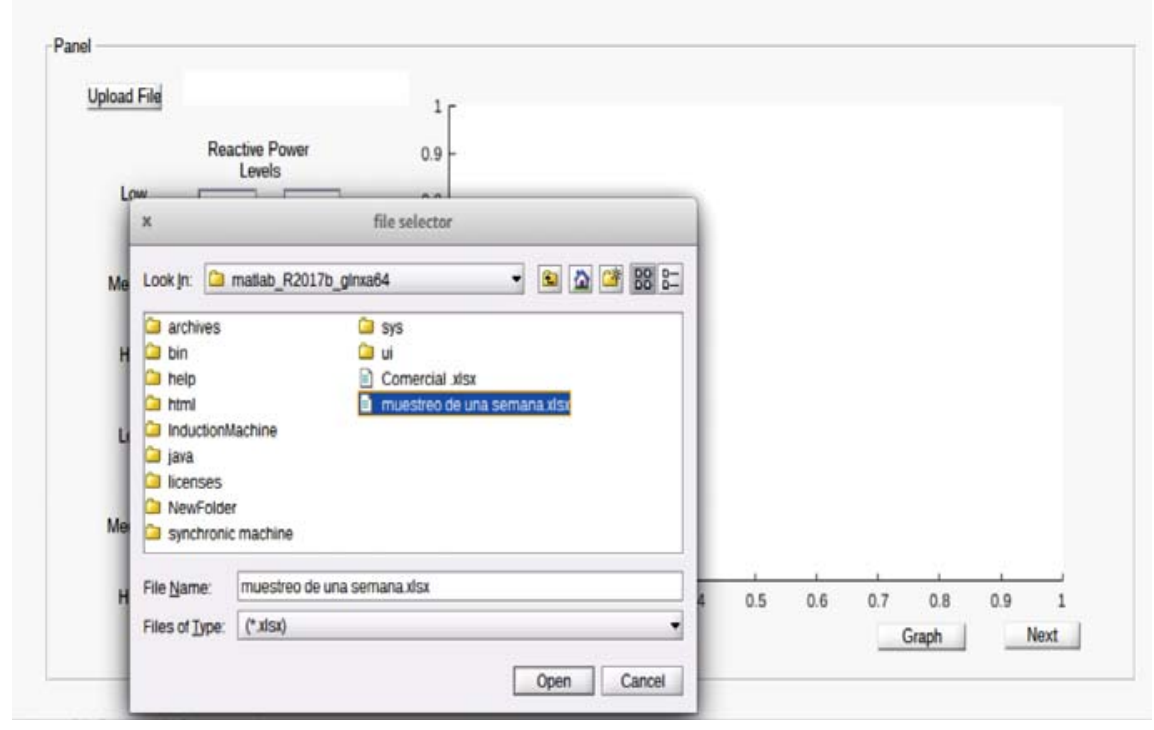

Fig. 2. Dataset opening window

The software divides the graphs into ranges to make the analysis more accurate. The designer must enter minimum, average, and maximum ranges of both initial PF and reactive power. The tool helps in this inspection process by showing the values corresponding to the position of the mouse pointer (Figs. 3 and 4). 


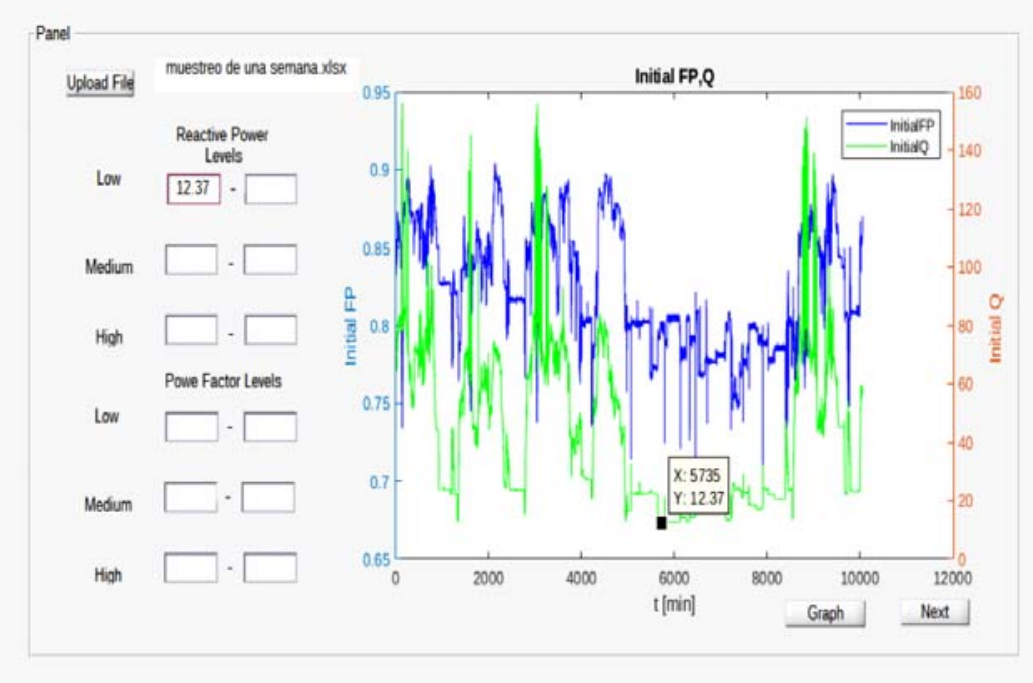

Fig. 3. Identification of the minimum values of $Q$

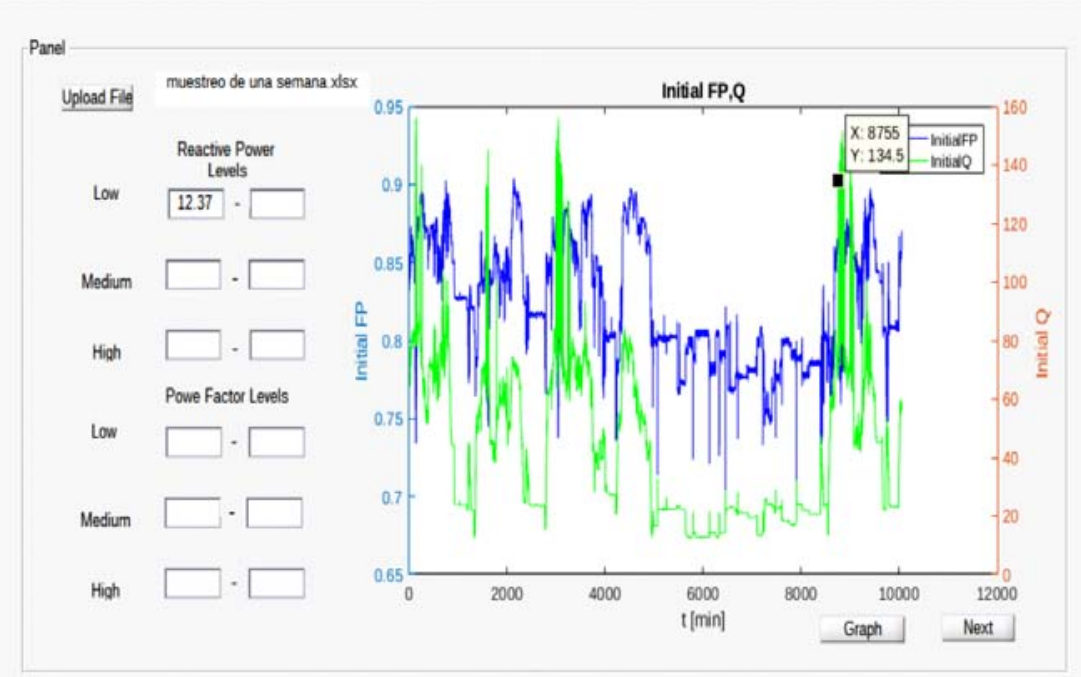

Fig. 4. Identification of the maximum values of $Q$

The reactive power ranges (low, medium and high) are essential for defining system conditions. The software proposes a set of values based on the analysis of the active power versus the reactive power of the system. The user can start the analysis from these values, or select others according to the behaviour observed in the graphs. These representations are important as it is not a question of analysing the reactive power in isolation, the level of correction depends on its relation to the active power. The active power is not constant over the sampling period.

It is also important to define the same values for PF (low, medium and high). Again the software proposes a set from the data, which can be adjusted by the designer according to his experience and behavioral observation (Fig. 5). 


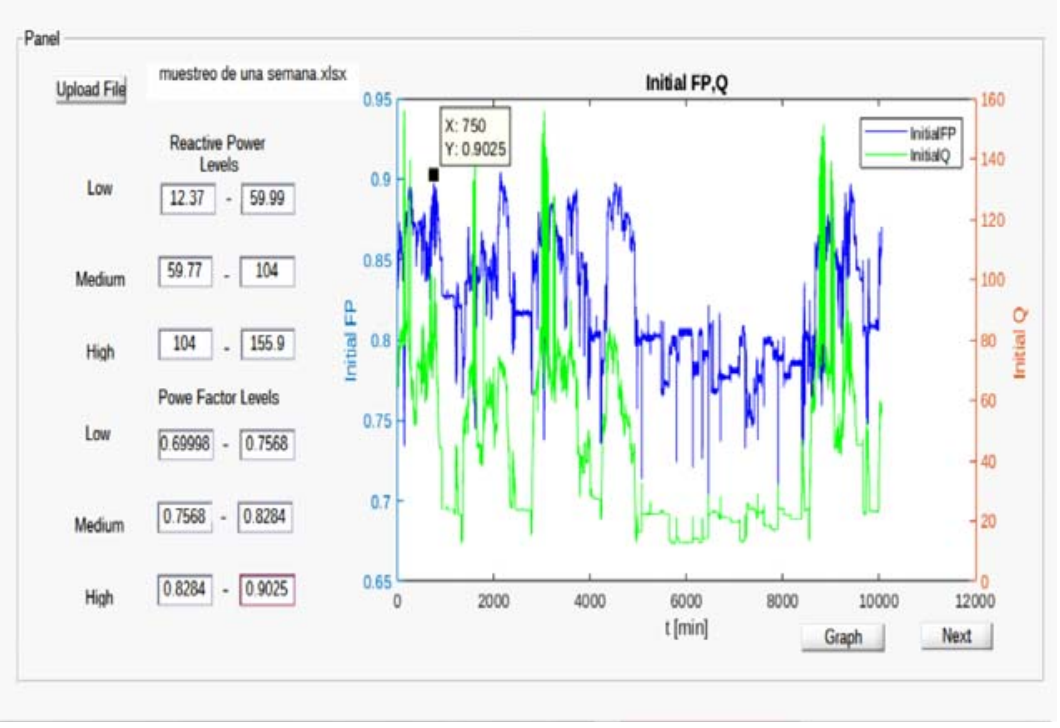

Fig. 5. Identification of the maximum values of PF

After fully selecting the intervals for both variables (Q and $\mathrm{PF}$ ), the program will independently select the most suitable kVAr capacitor bank, taking into account an acceptable PF range. The selection is made by means of a fuzzy inference machine. We selected two fuzzy inputs: PF and Q. For the fuzzy output variable PF we selected three fuzzy sets: Poor (P), Medium (M) and Acceptable (A) (Fig. 6). For the variable Q we normalize its value in relation to the active power in the range 0 to 1 , and evenly distribute three fuzzy sets: Low (L), Medium (M) and High $(\mathrm{H})$. The output variable is the reactive compensation power $\mathrm{Q}_{\mathrm{C}}$, which is normalised along the maximum potency present in the installation (Fig. 7).

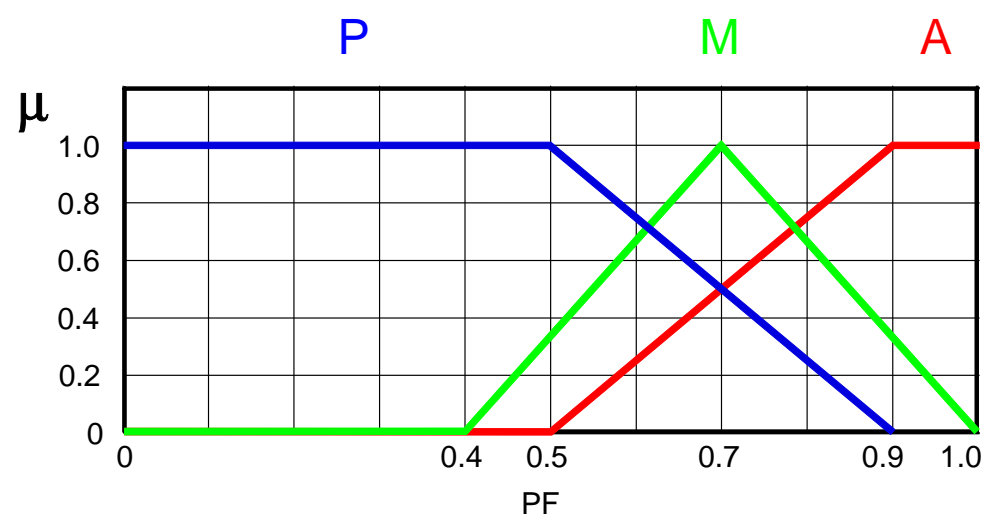

Fig. 6. Fuzzy sets for the fuzzy input variable PF
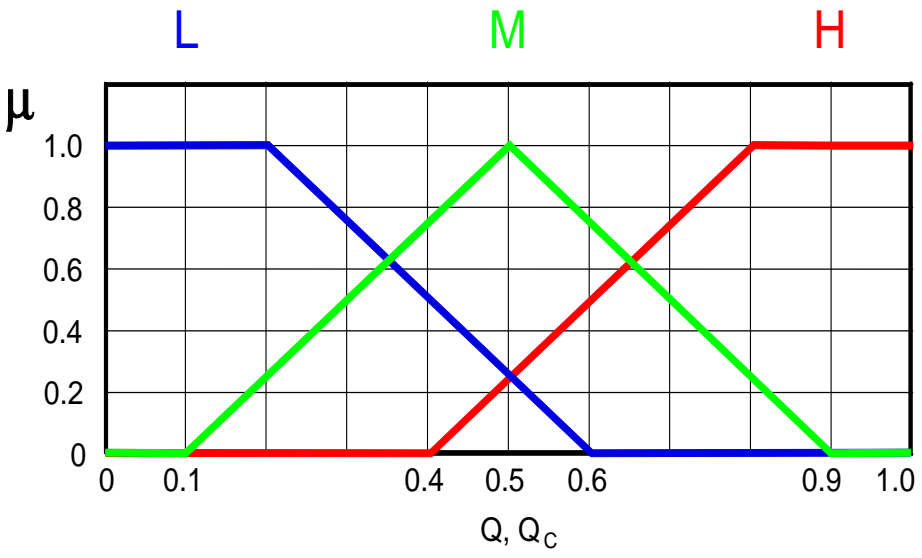

Fig. 7. Fuzzy sets for the fuzzy input variable $Q$ and fuzzy output variable $Q_{c}$

The choice is made from a database of commercial capacitor banks. The tool calculates approximately one capacitor bank per day, every 1440 data. Each day of operation forms a cycle; the software calculates the average values of $\mathrm{P}, \mathrm{Q}$ and $\mathrm{PF}$ for each cycle. The tool then determines whether these average values are in any 
of the previously defined low, medium, and high ranges. According to the location of the values, we apply the following fuzzy rules:

- If PF $€$ P AND Q $€$ L THEN $Q_{C} \in M$

- If PF $\in$ P AND Q $\in M$ THEN $Q_{C} \in H$

- If PF $\in$ P AND Q $\in$ H THEN $Q_{C} \in H$

- If PF $\in$ M AND Q $\in L$ THEN $Q_{C} \in L$

- If PF $\in M$ AND Q $\in M$ THEN $Q_{C} \in M$

- If PF $\in$ M AND Q $\in$ H THEN $Q_{C} \in H$

- If PF $€$ A AND Q $\in$ L THEN $Q_{C} \in L$

- If PF $€$ A AND Q $\in M$ THEN $Q_{C} \in L$

- If PF $€$ A AND Q $€$ H THEN Q $€$ $€$ L

Table 1 shows the values that make up the database of commercially available capacitor banks.

Table 1. Database of capacitor banks

\begin{tabular}{|c|}
\hline Capacity in kVAr \\
\hline 3 \\
\hline 6 \\
\hline 9 \\
\hline 12 \\
\hline 15 \\
\hline 18 \\
\hline 21 \\
\hline 24 \\
\hline 27 \\
\hline 30 \\
\hline 33 \\
\hline 36 \\
\hline 39 \\
\hline 42 \\
\hline 45 \\
\hline 48 \\
\hline 51 \\
\hline
\end{tabular}

\section{RESULTS AND DISCUSSION}

For the performance analysis of the tool we rely on a dataset captured in a real plant. We use a power analyzer to record power behaviour for one week, at one-minute intervals (dataset with 10,080 records for P, S, $\mathrm{VAr}$ and PF). The basic feature of the tool is its ability to visualize this information in a graphical way, initial step for the characterization of the problem (Fig. 8).

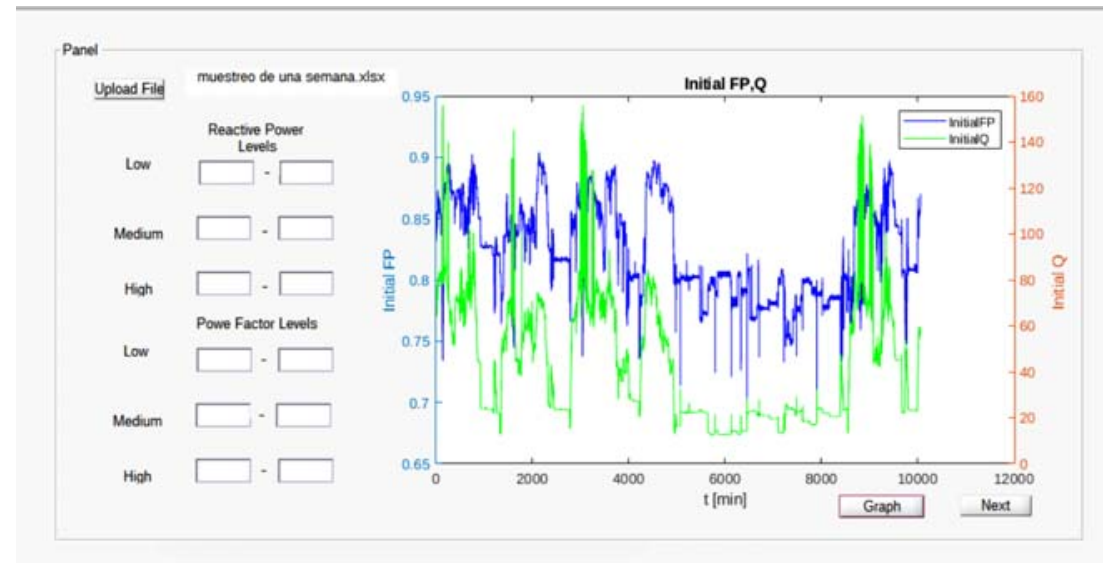

Fig. 8. Visualization of PF and Q from the dataset captured in tests 
For this particular case, the tool proposes minimum, average and maximum reactive power values (per day):

- Day 1: 24.6 kVAr, 54.4 kVAr and 77.6 kVAr

- Day 2: 25.6 kVAr, 54.4 kVAr and $74.9 \mathrm{kVAr}$

- Day 3: 36.6 kVAr, 71.6 kVAr and 129.4 kVAr

- Day 4: $20.3 \mathrm{kVAr}, 50.6 \mathrm{kVAr}$ and $73.9 \mathrm{kVAr}$

- Day 5: $13.4 \mathrm{kVAr}, 20.6 \mathrm{kVAr}$ and $22.8 \mathrm{kVAr}$

- Day 6: 21.2 kVAr, 35.6 kVAr and 55.6 kVAr

- Day 7: 24.0 kVAr, $50.1 \mathrm{kVAr}$ and $80.7 \mathrm{kVAr}$

In this design the proposed values have not been modified, they were validated as correct and we continue with the tool's proposals. According to this, the values selected for the six steps are:

- $12 \mathrm{kVAr}$

- $15 \mathrm{kVAr}$

- $21 \mathrm{kVAr}$

- $24 \mathrm{kVAr}$

- $30 \mathrm{kVAr}$

- $36 \mathrm{kVAr}$

By applying the switching rules for the selection of the reactive power, the appropriate step is selected. With this value the final behaviour of the corrected PF can be calculated (Fig. 9). This curve was calculated by applying the calculated capacitor bank to the data captured at the plant.

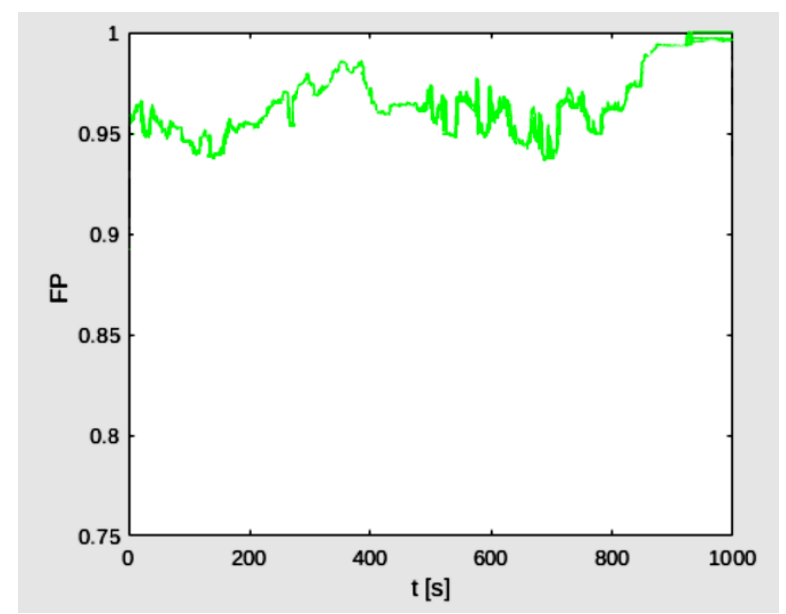

Fig. 9. PF corrected by applying the switching sequence for the selected six-step mixed compensation bank

This result confirms that the solution selected by the tool corrects the poor PF of the plant.

\section{CONCLUSION}

In this paper we present a software tool to support the design of solutions to poor displacement PF in industrial plants. The tool uses as input information the historical characteristic of sensed power in the plant's electrical installation. Based on this data, the software allows the graphical analysis of the information and proposes characteristic reactive power values for each of the plant's operating cycles. Then, using fuzzy inference, the tool defines from a configurable database the most suitable steps for the capacitor bank. The tool was successfully evaluated with real data from an industrial plant where it was able to identify a suitable solution.

\section{ACKNOWLEDGMENT}

This work was supported by the Universidad Distrital Francisco José de Caldas, in part through CIDC, and partly by the FacultadTecnológica. The views expressed in this paper are not necessarily endorsed by Universidad Distrital. The authors thank the research groups ARMOS and DIGITI for the evaluation carried out on prototypes of ideas and strategies. 


\section{REFERENCES}

[1] C. Heger, P. Sen and A. Morroni, Power factor correction - a fresh look into today's electrical systems, IEEE-IAS/PCA 54th Cement Industry Technical Conference, 2012, pp. 1-13.

[2] R. El-Mahayni, R. Vijver, R. Rashidi and A. Al-Nujaimi, Corporate wide power factor correction application: economic and technical assessment, Petroleum and Chemical Industry Conference Europe (PCIC Europe 2017), 2017, pp. 1-13.

[3] R. Pelzer, J. Swanepoel and J. Vosloo, Reactive power: A simplified model to justify the cost of interventions, Proceedings of the 9th Industrial and Commercial Use of Energy Conference, 2012, pp. 1-4.

[4] M. Khan and M. Owais, Automatic power factor correction unit, International Conference on Computing, Electronic and Electrical Engineering (ICE Cube 2016), 2016, pp. 283-288.

[5] D. Reljić, V. Vasić and D. Oros, Power factor correction and harmonics mitigation based on phase shifting approach, 15th International Power Electronics and Motion Control Conference (EPE/PEMC 2012), 2012, pp. DS3b.12-1-DS3b.12-8.

[6] N. Ramzan, A. Akbar, Z. Khan, P. Naseer and N. Zaffar, Reactive power compensation through synchronization of networked VFDs, Clemson University Power Systems Conference (PSC 2016), 2016, pp. 1-7.

[7] P. Almeida, A. Silva, M. Lemes and F. Garcia, Application of reactive compensation equipment in industrial systems under aspects of harmonics and switching transients: A study of real case, 17th International Conference on Harmonics and Quality of Power (ICHQP 2016), 2016, pp. 798-803.

[8] Y. Liu, S. Rau, C. Wu and W. Lee, Improvement of power quality by using advanced reactive power compensation, IEEE/IAS 52nd Industrial and Commercial Power Systems Technical Conference (I\&CPS 2016), 2016, pp. 1-6.

[9] M. Badr, M. Maarouf, M. Basyouni and S. Ahmed, Reducing harmonic distortion and correcting power factor in distribution systems, 22nd International Conference and Exhibition on Electricity Distribution (CIRED 2013), 2013, pp. 1-4.

[10] M. Deliu, S. Scridon, A. Hedes and N. Muntean, Practical aspects regarding power factor correction and harmonic mitigation of variable speed drives, 16th International Power Electronics and Motion Control Conference and Exposition, 2014, pp. 1289-1294.

[11] A. Maklakov, A. Radionov and V. Gasiyarov, Power factor correction and minimization THD in industrial grid via reversible medium voltage AC drives based on 3L-NPC AFE rectifiers, 42nd Annual Conference of the IEEE Industrial Electronics Society (IECON 2016), 2016, pp. 2551-2556.

[12] G. Costa, H. Fantoni, H. Tassara and I. Camargos, Harmonic mitigation techniques applied to industrial power systems: Real case study with measurements, SimposioBrasileiro de SistemasEletricos (SBSE 2018), 2018, pp. 1-6.

[13] K. Jeong, H. Lee, J. Park and Y. Baek, Optimal dispatch scheduling with synchronous machines for improving operational efficiency in industrial power system, IEEE International Conference on Smart Instrumentation, Measurement and Applications (ICSIMA 2013), 2013, pp. 1-6.

[14] J. Galvis and J. Madrid, Sistema de control difuso para motor de corriente continua sin escobillas (bldc) sobre hardware embebido, Tekhnê, vol. 13(2), 2016: pp. 43-48, ISSN 1692-8407.

[15] R. Jain, S. Sharma, M. Sreejeth and M. Singh, Plc based power factor correction of 3-phase induction motor, IEEE 1st International Conference on Power Electronics, Intelligent Control and Energy Systems (ICPEICES 2016), 2016, pp. 1-5.

[16] N. Kulkami, H. Murthy and A. Raju, Plc based intelligent power factor correctors for industrial power systems-a case study, International Conference on Power and Advanced Control Engineering (ICPACE 2015), 2015, pp. 75-79.

[17] A. Karimeh, N. Karami and H. El-Sheikh, A modified adaptive power factor correction technique, IEEE International Multidisciplinary Conference on Engineering Technology (IMCET 2016), 2016, pp. 97-102.

\section{AUTHOR PROFILE}

Fredy Hernán Martínez Sarmiento. PhD in Engineering - Systems and Computation, Professor at the Universidad Distrital Francisco José de Caldas, Director and principal researcher of the ARMOS research group.

Fernando Martínez Santa. MSc in Electronic and Computer Engineering, Professor at the Universidad DistritalFrancisco José de Caldas, Researcher of the ARMOS research group.

Edwar Jacinto Gómez. MSc inSciences of the Information and Communications, Professor at the Universidad Distrital Francisco José de Caldas, Researcher of the ARMOS research group. 